Article

\title{
Drying of a Colloidal Suspension Deposited on a Substrate: Experimental and Numerical Studies
}

\author{
Nathalie Olivi-Tran*(D), Laurent Bonnet and Pascal Etienne \\ Laboratoire Charles Coulomb, Universite de Montpellier, CNRS UMR 5221, CC 074, Place E.Bataillon, \\ F-34095 Montpellier, France; laurent.bonnet@umontpellier.fr (L.B.); pascal.etienne@umontpellier.fr (P.E.) \\ * Correspondence: nathalie.olivi-tran@umontpellier.fr
}

Citation: Olivi-Tran, N.; Bonnet, L.; Etienne, P. Drying of a Colloidal Suspension Deposited on a Substrate: Experimental and Numerical Studies. Crystals 2021, 11, 829. https:// doi.org/10.3390/cryst11070829

Academic Editor: Slobodan Žumer

Received: 30 June 2021

Accepted: 15 July 2021

Published: 17 July 2021

Publisher's Note: MDPI stays neutral with regard to jurisdictional claims in published maps and institutional affiliations.

Copyright: (c) 2021 by the authors. Licensee MDPI, Basel, Switzerland. This article is an open access article distributed under the terms and conditions of the Creative Commons Attribution (CC BY) license (https:// creativecommons.org/licenses/by/ $4.0 /)$.

\begin{abstract}
We studied a colloidal suspension of polystyrene beads deposited on a glass substrate. The glass substrate contained either straight rough areas on the borders of an open channel or only straight rough areas. The drying of the suspension was observed with an optical microscope, the light bulb of which acted as an energy source to evaporate the suspension. Moreover, the light bulb of the microscope provided optical pressure due to light. We observed that the colloidal particles were trapped on the rough areas of the substrate and not in the open channel at the end of the drying process. In order to understand the experimental results, we modeled numerically the drying of the suspension using a Molecular Dynamics program. The forces imposed on the substrate by the particles are their weight, the optical pressure due to the light bulb of the optical microscope, the attractive Van der Waals force and the repulsive diffuse layer force. The forces acting between two particles are the attractive Van der Waals forces, the repulsive diffuse layer force and the capillary force. The Gaussian random force (linked to Brownian motion) and the particle liquid viscous drag force (also linked to Brownian motion) are horizontal and applied on one particle. The relation between the normal forces $N$ (forces acting by the particles on the substrate) and the horizontal forces $F$ is Amontons' third law of friction $F \leq \mu_{k} N$; in rough areas of the substrate, $\mu_{k}$ is larger than in smooth areas. This explains that particles are trapped in the areas with high roughness.
\end{abstract}

Keywords: polystyrene beads; glass substrate; molecular dynamics; self patterning

\section{Introduction}

Since the beginning of the 21st century, microfluidics have attracted increasing interest. Among microfluidics studies, experiments and models of colloidal suspensions are becoming increasingly numerous. Many studies of colloidal suspensions deal with the self-patterning of the colloidal particles during drying. The patterns depend on many different factors: the size of the colloidal particles, chemical characteristics of the fluid ( $\mathrm{pH}$ and ionic concentrations), boundaries of the liquid suspension (if it is a droplet), densities of the colloidal suspension, the effect of temperature and the effect of the different forces acting on the particles. For example, Liang et al. [1] computed the forces (DLVO (DerjaguinLandau-Verwey-Overbeek) forces) which are applied on colloidal particles in a liquid. Bordin [2] modeled aggregation patterns (self-patterning) in a 2D model for colloids as a function of densities of the suspension and as a function of temperature. Ghosh et al. [3] studied the surface wettability of the substrate and its effect on the morphology of the deposited colloidal films. Bevan and Prieve [4] studied the Van der Waals attraction between the substrate and the particles or between the particles, as we also need to compute in our work. Finally, Thiele [5] used a mesoscopic hydrodynamic longwave model to explain self-pattering, and Giorgiutti-Dauphine et al. [6] studied the patterns obtained with the contact lines of water-air-particles at the boundary of the liquid film (if it is a droplet) and with nanometer-sized particles. The differences between these studies and our work are explained in the following paragraph. 
There is a need to model the process of the drying of dispersions in the domain of particles depositions correctly. Therefore, we study the drying of a liquid film of a colloidal suspension deposited on a substrate containing an open channel with rough areas on its borders or only straight and rough areas without any open channel under the light of a microscope light bulb. In order to obtain rough areas, we scratched these areas with a diamond pen. We assumed that the liquid film would have a surface which is planar and parallel to the substrate, and that the width of the film (horizontal size) would be much larger than the microscope light beam. The size of the colloidal spherical particles was chosen to be $1 \mu \mathrm{m}$. This size is intermediate between nanoscale physics and micrometer scale physics. This topography was only studied once (see reference [7]) but with a suspension of faceted particles with a size of $10 \mu \mathrm{m}$. Moreover, in [7], the numerical modeling did not take into account all the forces used in this present study. Indeed, several forces act between the particles and the substrate and between the particles themselves: the first of these are the forces resulting from microfluidics: capillary forces $[7,8]$, Brownian forces and Stokes forces [9]; the second are the DLVO forces: van der Waals forces [3] and electrostatic double layer forces [10]; the third are the optical forces [11] which result from the irradiance of the light bulb of the microscope; the fourth are the friction forces, which differ depending on where the particles are located-in the rough areas of the substrate or in the smooth areas of the substrate-and the fifth force is the weight of the particles. Plus, the $p H$ of the suspension was neutral and the temperature was constant, and we studied two suspension densities.

Experimentally, after the drying of the colloidal suspension, the particles were mainly located on the rough parts of the substrate. Even when the open channels were deeper than the diameter of the particles, these particles did not stay in these channels once all the liquid was evaporated (except for a few particles).

Numerically, we computed all the forces acting on the particles. To date, no numerical study of the drying of a colloidal suspension film has dealt with all the forces mentioned above.

In Section 2, we present the experimental process of the drying of a liquid suspension of colloidal particles. Section 3 deals with the numerical method employed (Molecular Dynamics). Finally, Section 4 shows the experimental and numerical results, with a comparison between them.

\section{Experimental Processes}

We study the pattern formation of spherical colloidal particles in an aqueous suspension when the suspension is dried on a flat substrate with defects. The defects are rough areas of the substrate and micrometer-sized open channels.

The experimental suspension is made of dark red 5\%wt micro-particles based on polystyrene (PS) in an aqueous suspension (Sigma Aldrich). The particles are spherical colloidal particles with a mean diameter of $d=1 \mu \mathrm{m}$. We used two different dilutions of the initial particle concentration: the first diluted suspension was obtained by adding $1.5 \mathrm{~cm}^{3}$ of deionized water to $0.05 \mathrm{~cm}^{3}$ of colloidal suspension, while the second diluted suspension was obtained by adding $3 \mathrm{~cm}^{3}$ of deionized water to $0.05 \mathrm{~cm}^{3}$ of colloidal suspension. For the first diluted suspension, the number of particles per volume unit was equal to $8.5 \times 10^{10} \pm 0.05 \times 10^{10} \mathrm{~cm}^{-3}$ for the second diluted suspension, the number of particles per volume unit was equal to $1.67 \times 10^{9} \pm 0.03 \times 10^{9} \mathrm{~cm}^{-3}$. The $p H$ of the suspension was equal to 7 .

We deposit the aqueous suspension on a microscope slide (a Borosilicate glass substrate with dimensions $75 \mathrm{~mm} \times 25 \mathrm{~mm} \times 1 \mathrm{~mm}$ ). The suspension was extended on the glass substrate and we waited $20 \mathrm{~min}$ in order for the depth of the suspension to be homogeneous. We obtained a liquid film of $5 \pm 0.5 \mu \mathrm{m}$ in depth.

The surface roughness of the PS particles is plotted in Figure 1a. This surface roughness is an AFM (Atomic Force Microscope) scan obtained with the Nanosurf Nanite AFM apparatus. The surface roughness of the glass substrate is plotted in Figure $1 \mathrm{~b}$ and is obtained with the same apparatus (AFM) as the surface roughness of the PS particles. 
The smaller asperities of the spherical particles have a height (peak-to-valley) of $20 \mathrm{~nm}$ with a width of $10 \mathrm{~nm}$ (Figure 1a). The smaller asperities of the glass substrate have a height (peak-to-valley) of $20 \mathrm{~nm}$ with a width of $2 \mu \mathrm{m}$ (Figure $1 \mathrm{~b}$ ). In order to obtain the distance between the particles and the substrate, we followed the same process as in the article of Bevan [4] (the roughnesses of the glass and of PS particles are similar to Bevan's results): the dielectric properties of the liquid film were those of the fluid (water) and the thickness of the liquid film (between the particle and the substrate) corresponded to the peak-to-valley distance $(20 \mathrm{~nm})$. Put simply, the smallest distance (surface to surface) between the particles and the substrate was set to be equal to $20 \mathrm{~nm}$ and the smallest distance between a particle and another particle was also set to be equal to $20 \mathrm{~nm}$. This was used in the numerical modeling of the drying of the suspension.

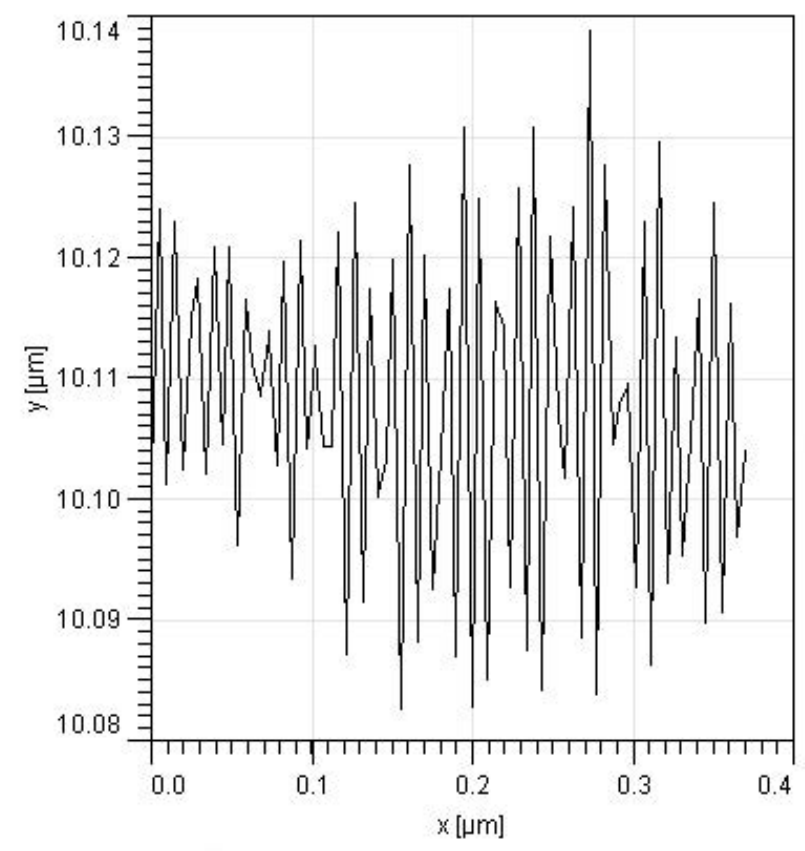

(a)

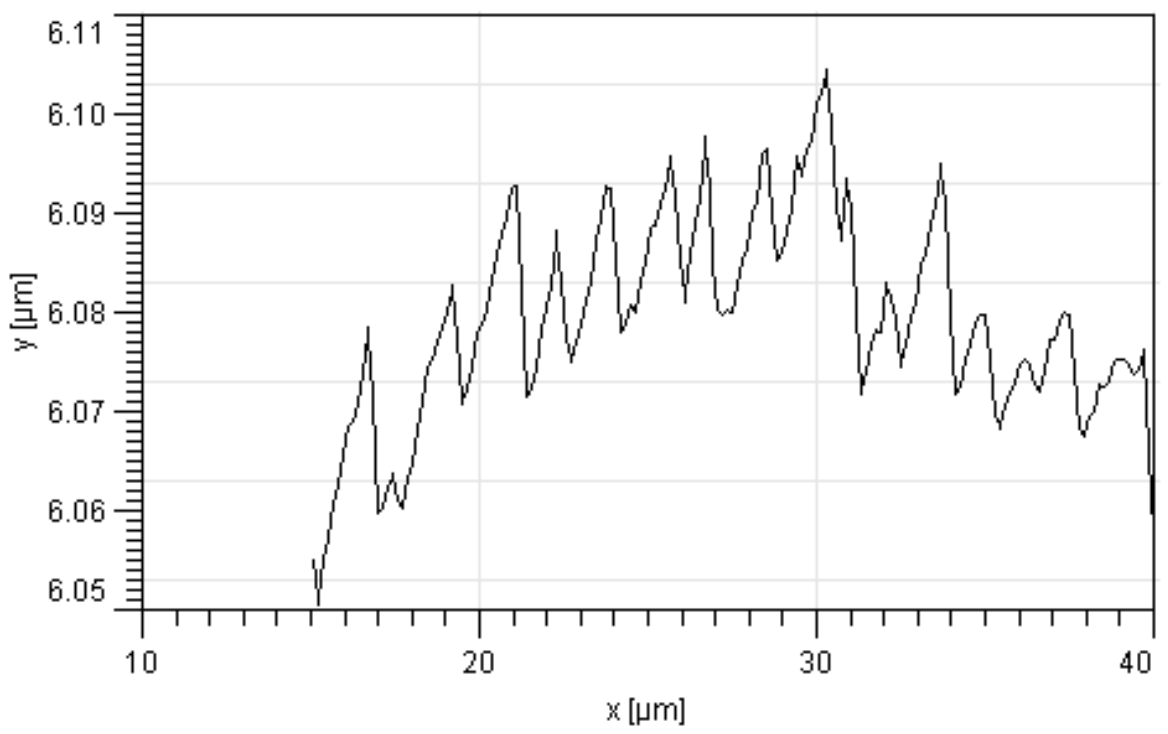

(b)

Figure 1. (a) AFM scan of the surface roughness of a colloidal particle of PS, (b) AFM scan of the surface roughness of the microscope slide-substrate. 
We used an optical microscope (Keyence HTX 7000) in order to visualize the colloidal particles on their substrate. These substrates were scratched with a diamond pen, either with a light pressure or with a larger pressure of the pen on the slide. Figures 2 and 3 represent the two types of defects. Figure 2 shows an open channel with a width of $350 \pm 5 \mu \mathrm{m}$ and depth of $35 \pm 3 \mu \mathrm{m}$ obtained with a large pressure of the diamond pen. As may be seen in Figure 2, the borders of the channel were higher (red area) and could be modeled numerically by an open channel with rough borders. We used the more concentrated suspension on this substrate $\left(8.5 \times 10^{10} \mathrm{~cm}^{-3}\right)$. Figure 3 shows three stripes obtained with a light pressure of the diamond pen. These three stripes had a depth and a width of $1 \pm 0.2 \mu \mathrm{m}$ each and could be modeled by a straight linear area of width $1 \pm 0.2 \mu \mathrm{m}$, where the roughness was much larger than in the flat and smooth areas of the substrate. In this last case, there was no open channel, with only rough areas, and we used the less concentrated suspension on this substrate $\left(1.67 \times 10^{9} \mathrm{~cm}^{-3}\right)$.

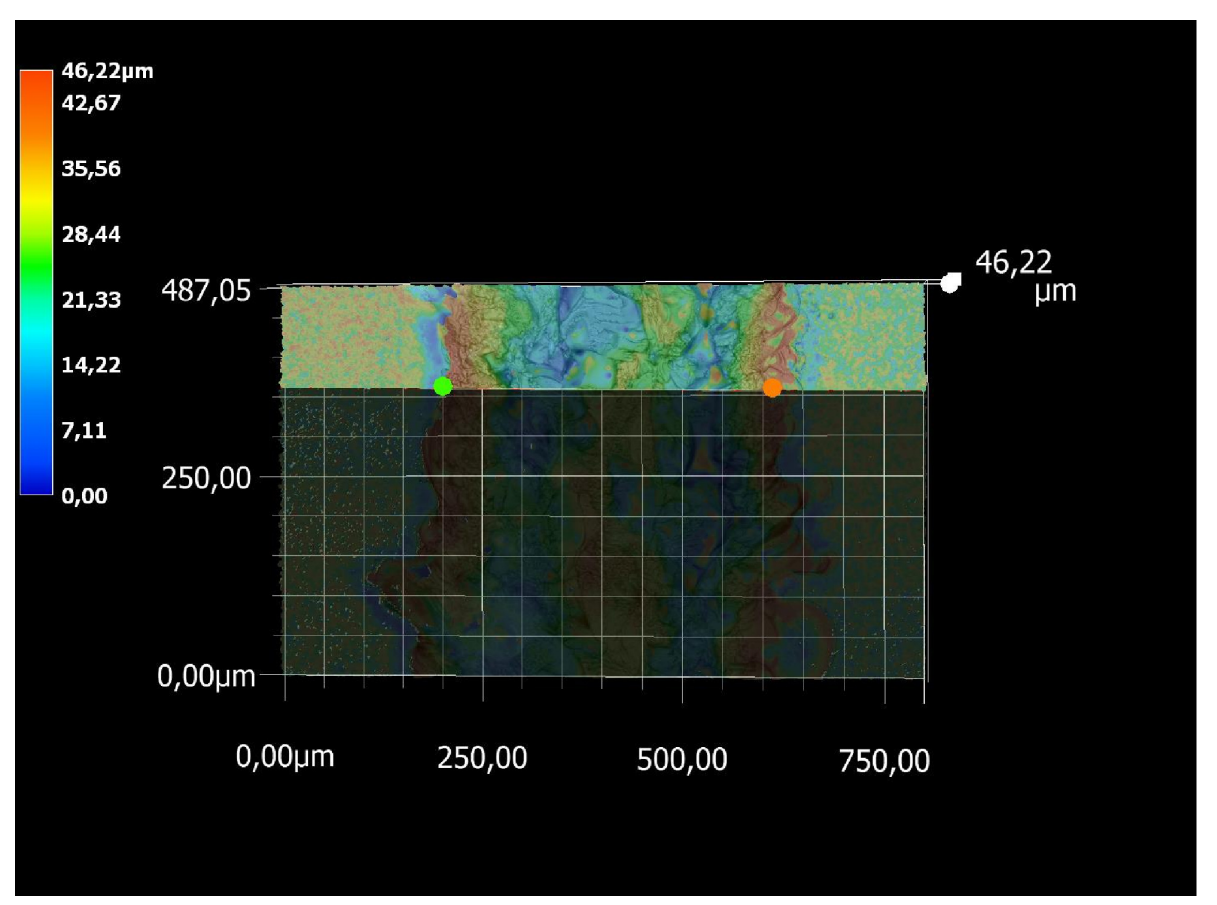

Figure 2. Top view obtained with an optical microscope of the substrate scratched with a large pressure (surface mapping). The stripe (open channel) had a width of $350 \mu \mathrm{m}$ and a depth of $35 \mu \mathrm{m}$. The borders of the stripe were higher and presented a roughness larger than the non-scratched areas of the substrate.

The microscope was equipped with a light bulb comprising an LED with a spectral irradiance as a function of wavelength plotted in Figure 4 [12]. The light bulb acted on the colloidal suspension in two ways: first, the evaporation of the water composing the suspension due to the power of light (coming from the light bulb and with a value of 10 Watts); second, the optical pressure applied by the light on the colloidal particles. The width of the liquid film was much larger than the width of the optical beam, and thus we did not take into account the contact line on the borders of the liquid film. Finally, the substrate remained horizontal, and the upper surface of the liquid film was also horizontal and parallel to the substrate. 


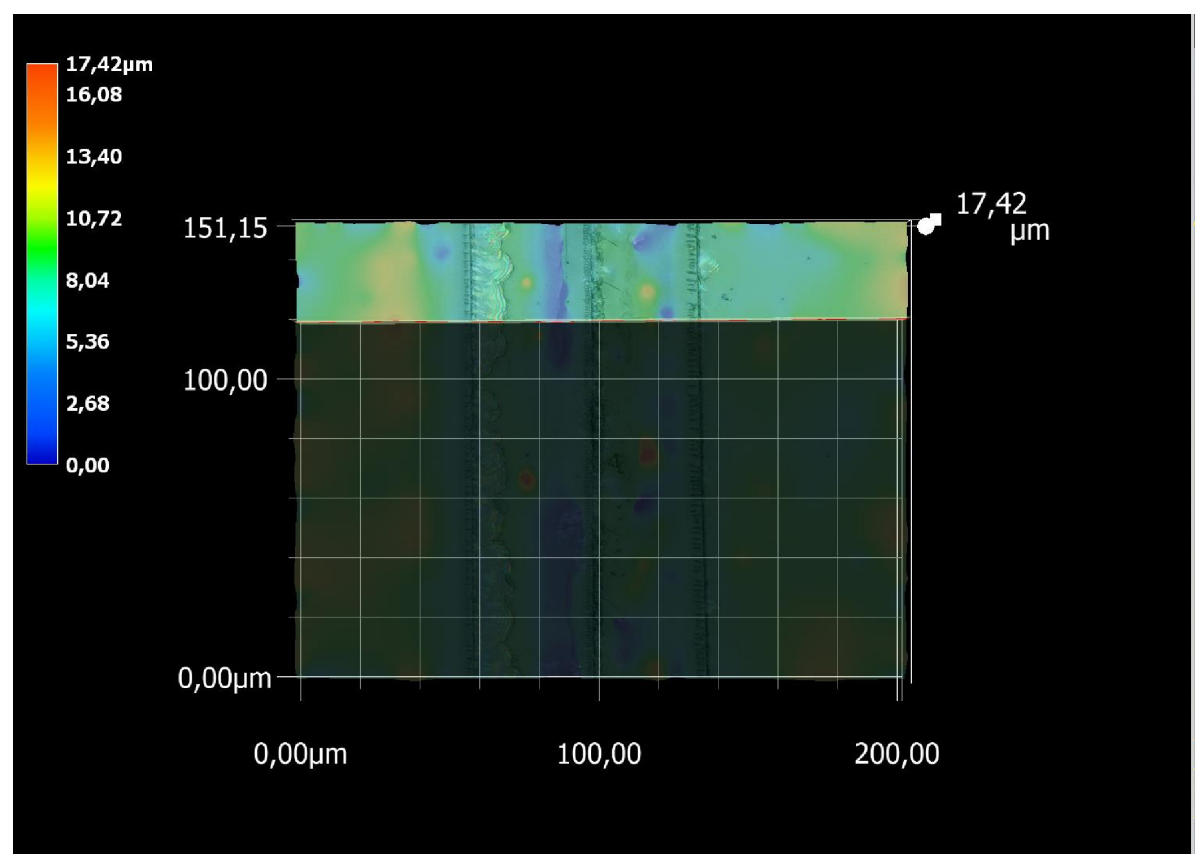

Figure 3. Top view obtained with an optical microscope of the substrate scratched with a light pressure (surface mapping). There were 3 straight areas of width $1 \mu \mathrm{m}$ and depth $1 \mu \mathrm{m}$, where the roughness was larger than the non-scratched areas.

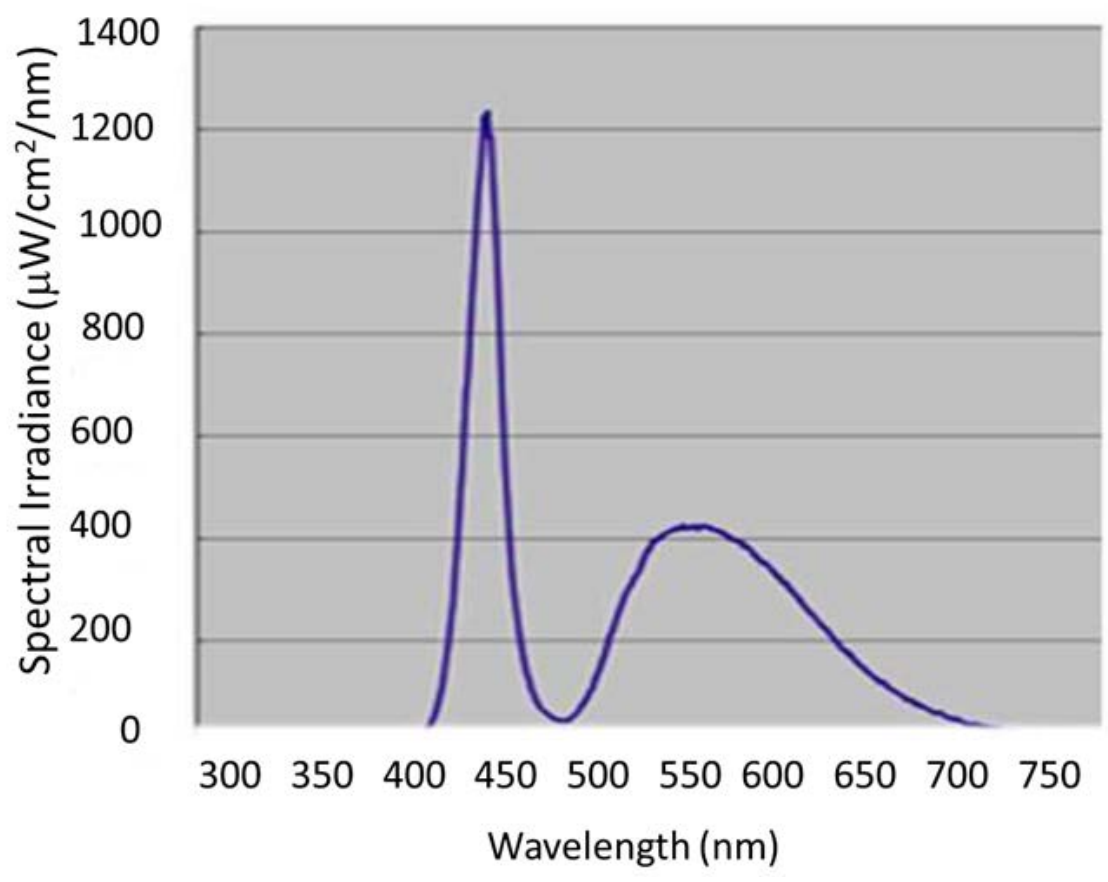

Figure 4. Spectral irradiance of the light coming from the light bulb of the optical microscope [12].

\section{Numerical Model}

We modeled the drying of a colloidal suspension over a plane substrate numerically with rough and smooth areas and with an open channel with Molecular Dynamics. The Molecular Dynamics program uses a predictor corrector method in order to compute forces, accelerations, velocities and positions with respect to time. The Molecular Dynamics simulations were performed at a constant temperature (330 K) to solve Newton's equation of motion for each particle. Hence, the trajectories of the particles were computed during the drying process of the liquid film, taking account of the fact that the depth $h$ of the film decreased. The integration time step was $\Delta t=10^{-7} \mathrm{~s}$. 
In order to fit the experimental data, the parameters employed in the numerical model were the same as the experimental parameters (see Table 1).

The thin film was numerically modeled by a collection of $N=600$ spherical particles of equal diameter $d=1 \mu \mathrm{m}$ and mass $m$, randomly distributed in a box of length $50 d$ in the $x$ and $y$ directions and of height $h=d / 2$ in the $z$ direction at the beginning of the computation. On the flat part of the substrate, the particles moved only in the $x$ and $y$ directions; if the particles were placed in the open channel, they were allowed to move also in the $z$ direction (the numerical process began when the thickness of the liquid film was equal to the diameter of the colloidal particles). In order to simplify the model, we developed the hypothesis that the particles would not roll but only slide over the substrate. To compute the effect of the liquid film on the particles, the depth $h$ of this film decreased linearly from $h=d / 2$ to $h=-10 d$, which was the depth of the open channel, or from $h=d / 2$ to $h=0$ when there was no channel. The air-liquid interface was characterized by $(x, y, h)$ while the liquid-solid interface was characterized by $(x, y, 0)$ on the flat part of the substrate or by $(x, y,-10 d)$ within the channel. Periodic boundary conditions were applied in the $x$ and $y$ directions because we did not take into account the contact lines of the liquid film and the substrate.

Below, we compute the different forces which were applied on the particles (the parameters and constants which are used to compute these forces are listed in Table 1).

\subsection{Vertical Forces}

As the particles are identical, their weights were equal.

The optical pressure, which models the effect of the white light (coming from the optical microscope bulb) on the particles, was also constant and identical for all particles. With the use of Figure 4, we can calculate the optical pressure induced by the light of the light bulb within the microscope. The surface area of the shadow (created by the colloidal particle of diameter $d$ ) is $S=\pi(d / 2)^{2}=\pi d^{2} / 4=\pi 10^{-12} / 4$. Simply, the estimated value of the optical force on one spherical particle can be written as follows $[13,14]$ :

$$
f_{o p t}=n_{r} \cdot I_{f} \cdot S / c
$$

where $I_{f}$ is the maximum of the spectral irradiance of the light bulb and $c$ is the speed of light (see Figure 4).

The attractive Van der Waals forces between the particles and the plane substrate can be written as follows [15]:

$$
f_{i}^{v d W p s}=-\left(d A_{132}\right) /\left(6(r-d)^{2}\right)
$$

where $r$ is the center-to-center distance between two colloidal particles $(r-d$ is always larger than $20 \mathrm{~nm}$; see Section 2) and $A_{132}$ is the Hamaker constant (PS-water-glass).

The repulsive force between the spherical particles and the plane substrate are [16]

$$
f_{i}^{e p s}=\kappa \epsilon d \pi\left(\phi_{1}^{2}+\phi_{2}^{2}-2 \phi_{1} \phi_{2} \exp (-\kappa(r-d)) /(\exp (-2 \kappa(r-d))+1)\right.
$$

where $\kappa$ is the Debye Huckel length, $\epsilon$ is the permittivity of water, $\phi_{1}$ is the surface potential of PS and $\phi_{2}$ is the surface potential of glass. This force is electrostatic.

\subsection{Horizontal Forces}

There is no shear flow of the colloidal suspension (the evaporation of the fluid leads to a homogeneous decrease of the fluid height $h$ all over the sample). In any case, the fluid is in the laminar state, and we focus on the regime of small Peclet numbers (the ratio of shear driven to Brownian motion at the particle scale) and we assume that the Reynold's number of the fluid-water-is small; thus, there is no convection. 
The particles are submitted to attractive Van der Waals forces (forces between two particles), which can be written as follows [10] ( $r-d$ is always larger than $20 \mathrm{~nm}$; see Section 2):

$$
f_{i}^{v d W p p}=-\left(d A_{131}\right) /\left(24(r-d)^{2}\right)
$$

where $A_{131}$ is the Hamaker constant (PS-water-PS). The particles are also submitted to repulsive electrostatic forces (forces between two particles) which can be written as follows [16]:

$$
f_{i}^{e p p}=\kappa \epsilon(d / 2)\left(\phi_{1}\right)^{2}(\exp (-\kappa(r-d)) /(1+\exp (-2 \kappa(r-d)))
$$

Particles that are not located in the channel but on the flat part (smooth or rough) of the substrate are subjected to lateral capillary forces (forces between two particles). These forces are attractive and are written as follows [7,8,17]:

$$
f_{i}^{c}(r)=-\pi \gamma r_{c}^{2} \sin \left(\phi_{c}\right)^{2} \frac{d}{(r-d)^{2}}
$$

where $r_{c}$ is the radius of the liquid-solid contact line on the spherical particles, $\phi_{c}$ is the mean slope angle of the meniscus at the contact line (also on the spherical particles), $\gamma$ is the surface tension of water and $R=d / 2$. Figure 5 shows a schematic description of these parameters. The analytical expressions of $r_{c}$ and $\phi_{c}$ are written as follows [7,8,17]:

$$
\begin{gathered}
r_{c}=(h(d-h))^{1 / 2} \\
\phi_{c}=\arcsin \left(\frac{2 r_{c}}{d}\right)-\alpha
\end{gathered}
$$

where $\alpha$ is the wetting angle at the three contact line (liquid-particle-air).

The particles are submitted to Brownian motion, which is linked to a viscous drag force according to the fluctuation-dissipation theorem. Thus, to compute the Brownian motion, we use a Gaussian random force which is expressed through the formalism of Wiener $[7,9]$ :

$$
f_{i}^{b}(t)=\sqrt{6 \pi k_{B} T h \eta} \Delta W(t)
$$

where $\Delta W(t)$ is computed with a Gaussian random number as $<\Delta W(t) \geq 0$ and $<\Delta W(t) \Delta W\left(t^{\prime}\right) \geq \Delta t$. This force is applied on one particle by the surrounding fluid and depends on $h$. Here, $k_{B}$ is the Boltzmann constant, $T$ the temperature and $\eta$ is the viscosity of water.

The particle liquid viscous drag force is computed with the Stokes formalism $[7,9]$ :

$$
f_{i}^{S}(t)=-3 \pi h \eta v_{i}(t)
$$

where $v_{i}(t)$ is the speed of the particle. This force is also applied on one particle by the surrounding fluid and depends also on $h$.

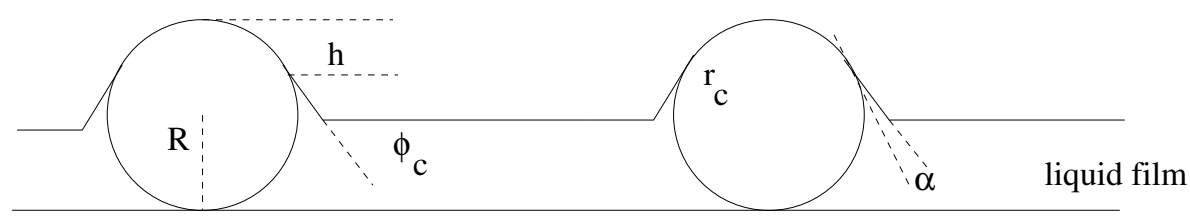

substrate

Figure 5. Scheme of a capillary bridge with all the parameters used in the numerical calculations $(R=d / 2)$.

\subsection{Relation between Horizontal and Vertical Forces}

Finally, we deal with the friction force induced by the roughness of the substrate. We present the hypothesis that the particles do not roll but only slide on the substrate and thus are submitted to friction forces. The vertical forces $N$ applied on the particles are the 
weight, the optical pressure, the van der Waals force and the diffuse layer (electrostatic) force. Simply, the kinetic friction force is opposed to the horizontal component of the forces applied on the particles as long as the sum of the horizontal forces $f_{i}^{h}$ obeys the following equation (Coulomb's law of friction, also called Amontons' third law):

$$
\left|f_{i}^{h}(r)\right| \leq \mu_{k} N
$$

where $\mu_{k}$ is the kinetic friction coefficient (for particles in motion parallel to the substrate) which is multiplied by the normal force $N$ (obtained with the weight, the optical pressure, the van der Waals force and the diffuse layer force between particles and substrate). There is no literature on the values of the kinetic friction coefficients that simulate the friction of PS on smooth areas of glass or that simulate the friction of PS on rough areas of glass. Therefore, we chose a large value (see Table 1) of $\mu_{k}$ for rough areas and a small value for smooth areas.

Table 1. Parameters and constants used in the numerical simulations.

\begin{tabular}{ccc}
\hline Name & Symbol & Value \\
\hline Density of PS & $\rho$ & 1.05 \\
Refractive index of PS & $n_{r}$ & 1.6 \\
Length side of simulation box & $\ell$ & $50 \mu \mathrm{m}$ \\
Particle diameter & $d$ & $1 \mu \mathrm{m}$ \\
Thickness of liquid film & $h$ & $d / 2$ to $-10 \mathrm{~d} \mu \mathrm{m}$ \\
Number of particles & $n_{1}$ & 600 \\
Debye Huckel length & $\kappa^{-1}$ & $\left(430.10^{-9}\right)^{-1} \mathrm{~m}^{-1}[18]$ \\
Permittivity of water & $\epsilon$ & 78.43 \\
Surface potential of PS & $\phi_{1}$ & $15 \mathrm{mV}[18]$ \\
Surface potential of glass & $\phi_{2}$ & $-40 \mathrm{mV} \mathrm{[18]}$ \\
Hamaker coefficient & $A_{131}$ & $3.10^{-20} \mathrm{~J} \mathrm{[19]}$ \\
(PS-water-PS) & $A_{132}$ & $10^{-20} \mathrm{~J} \mathrm{[18]}$ \\
Hamaker coefficient & & \\
(PS-water-glass) & $g$ & $9.81 \mathrm{~m} \cdot \mathrm{s}^{-2}$ \\
Gravitational acceleration & $\Delta t$ & $10^{-7} \mathrm{~s}$ \\
Time step & $\gamma$ & $73 \times 10^{-3} \mathrm{~N} \cdot \mathrm{m}^{-1}$ \\
Surface tension of water & $\alpha$ & $0.7 \mathrm{rad} \mathrm{[7]}$ \\
Wetting angle at three contact line & $T$ & $330 \mathrm{~K}$ \\
Temperature & $k_{B}$ & $1.3806 \times 10^{-23} \mathrm{~m}^{2} \cdot \mathrm{kg} \cdot \mathrm{s}^{-2} \cdot \mathrm{K}^{-1}$ \\
Boltzmann constant & $\eta$ & $0.5 \times 10^{-3} \mathrm{~kg} \cdot \mathrm{m}^{-1} \cdot \mathrm{s}^{-1}$ \\
Viscosity & $\mu_{k}$ & 0.9 \\
Kinetic friction coefficient & $\mu_{k}$ & 0.3 \\
Rough areas of substrate & & \\
Kinetic friction coefficient & & \\
Smooth areas of substrate & &
\end{tabular}

When Equation (11) is valid, one has to add a friction force to all the forces that act on a particle (this friction force has a norm equal to $\left|f_{i}^{h}(r)\right|$ with an opposite direction). For large roughnesses $\left(\mu_{k}=0.9\right.$, see Table 1$)$, the horizontal forces applied on one particle are multiplied by 0.1 ; thus, the displacement of the particle is small in this case, and the particle remains close to the rough area. In the case of smooth areas $\left(\mu_{k}=0.3\right.$, see Table 1$)$, the horizontal forces are multiplied by 0.7 , and thus the particle can move on these areas (larger displacements). When Equation (11) is not valid, the horizontal forces remain unchanged for rough and smooth areas (we do not add the friction force to all the forces applied to one particle). 


\section{Results and Discussion}

The experimental results that show the patterns obtained when the colloidal suspension is dried are represented in Figures 6 and 7. By comparing the substrate with rough areas and with an open channel (Figure 2) with the equivalent substrate with the dried colloidal suspension (Figure 6), one may see that particles are trapped in the rough areas but not in the open channel (except a few particles). Similarly, by comparing the substrate with rough areas (Figure 3) and without an open channel with the dried suspension on the equivalent substrate, one may see again that the particles remain in locations where the roughness is higher (Figure 7). Figure 3 has fewer rough areas than Figure 7, but both have rough, straight areas in which particles are trapped (the number of stripes obtained with a light pressure of the diamond pen depends on the wear of the pen). In Figures 6 and 7, particles that are not located in the rough areas are randomly distributed in the smooth areas of the substrate. In order to understand the mechanisms which lead to these results, we modeled the drying of the colloidal suspensions on substrates with a large channel and/or rough areas. Thus, we used a Molecular Dynamics program.

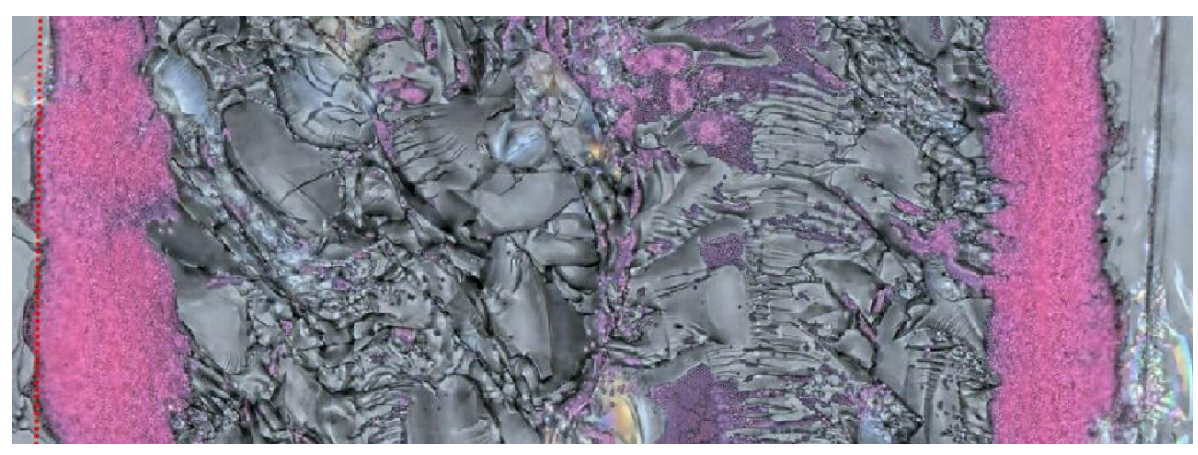

Figure 6. Top view of a dried liquid film on a substrate containing an open channel bordered by two rough areas. The colloidal particles are trapped on the rough parts of the substrate.

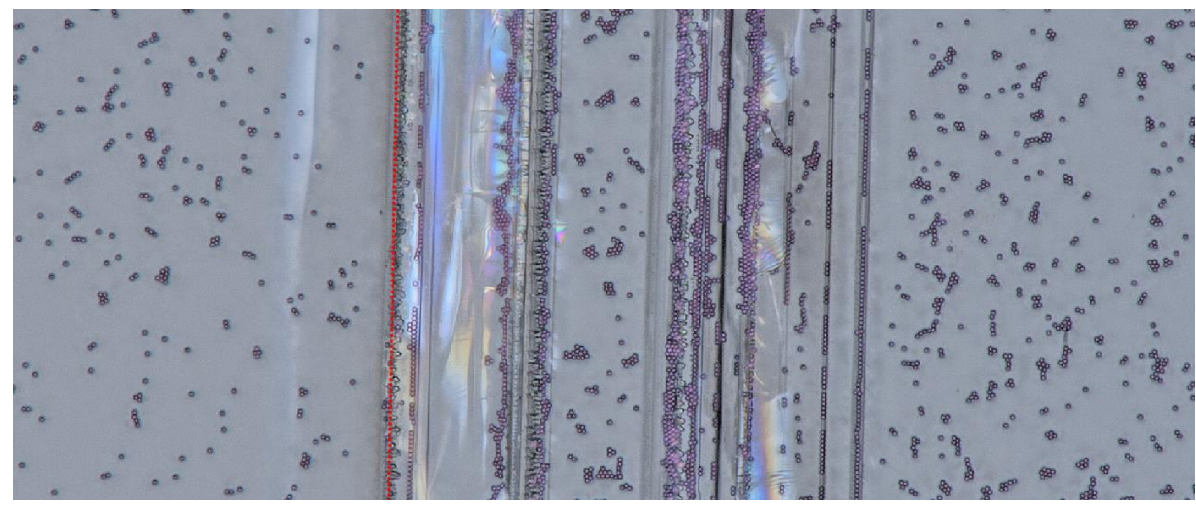

Figure 7. Top view of a dried liquid film on a substrate with several rough and straight areas of the substrate. The colloidal particles are mainly located on the straight and rough areas.

The weight of each spherical particle is equal to $4 / 3 \pi(d / 2)^{3} . \rho . g=5.39 \times 10^{-18} \mathrm{~N}$ (see Table 1 for the values and definition of the parameters).

The maximum of $I_{f}$ (spectral irradiance) is equal to $1200 \times 10^{7} \mathrm{~W} / \mathrm{m}^{3}$ (see Figure 4). Thus, the value of the optical force is $1200 \times 10^{7}(\pi / 4) 10^{-12} /\left(3.10^{8}\right)=5.02 \times 10^{-11} \mathrm{~N}$ for each particle (see Equation (1)).

The value of the attractive van der Waals force between the particle and the substrate is calculated with Equation (2) and yields the value of $f_{v d w}=1.25 \times 10^{-11} \mathrm{~N}$. The value of the repulsive force due to the electrostatic diffuse layer between the particle and the substrate is calculated with Equation (3); this force is equal to $1.13 \times 10^{-11} \mathrm{~N}$ (assuming that the surface to surface distance is equal to $20 \mathrm{~nm}$, see Figure 1a,b and Section 2). 
Thus, the normal forces applied on the substrate by a particle are obtained by adding all the attractive forces and subtracting the repulsive forces calculated above; these equal $5.14 \times 10^{-11} \mathrm{~N}$.

In order to model the effect of friction on the patterning of the colloidal particles, we computed all the horizontal forces acting on these particles (see Figures 8 and 9). One may see in Figure 8 that capillary forces and electrostatic diffuse layer forces are dominating (for the case where there is an open channel in the substrate). However, in Figure 9 (for the case where there are only rough straight areas in the substrate), only capillary forces are dominating. This is explained by the fact that particles that are located in the open channel are not subject to capillary forces (particles are completely surrounded by the fluid, with a depth $0>h>-10 d)$ ), except at the end of the drying process, so the sum of all the capillary forces is smaller when there is an open channel. This can also be deduced from the comparison between Figures 8e and 9e: the capillary forces differ from a factor of 10.

In the computation, if the absolute value of the addition of all horizontal forces applied on one particle obeys Amontons' third law (Coulomb's law), and if the particle is located in a rough area of the substrate, the horizontal forces applied on this particle are multiplied by 0.1. Simply, the probability for one particle to be trapped in its location is much larger when the kinetic friction coefficient is large (rough areas) than when the kinetic friction coefficient is small (in that case, the horizontal forces applied on this particle are multiplied by 0.7). The results are shown in Figures 10 and 11. One may see that most of the particles are trapped in the rough areas in both cases (with or without open channel) and the patterns obtained numerically are the same as the patterns obtained experimentally.

We modeled the effect of evaporation by decreasing linearly the depth of the liquid film $h$ with time $t$.The effect of evaporation in Equations (6)-(8), which are functions of the depth of the liquid film $h$, is shown in lateral capillary forces using $r_{c}\left(r_{c}\right.$ is the radius of the liquid solid contact line on one particle), $\phi_{c}$ (which is the mean slope angle of the meniscus at the contact line on one particle) and $\alpha$ (which is the wetting angle at the three contact line (liquid-particle-air)). The particle liquid viscous drag force acting on one particle (Equation (10)) also depends on $h$ as well as the Gaussian random force (Equation (9)).

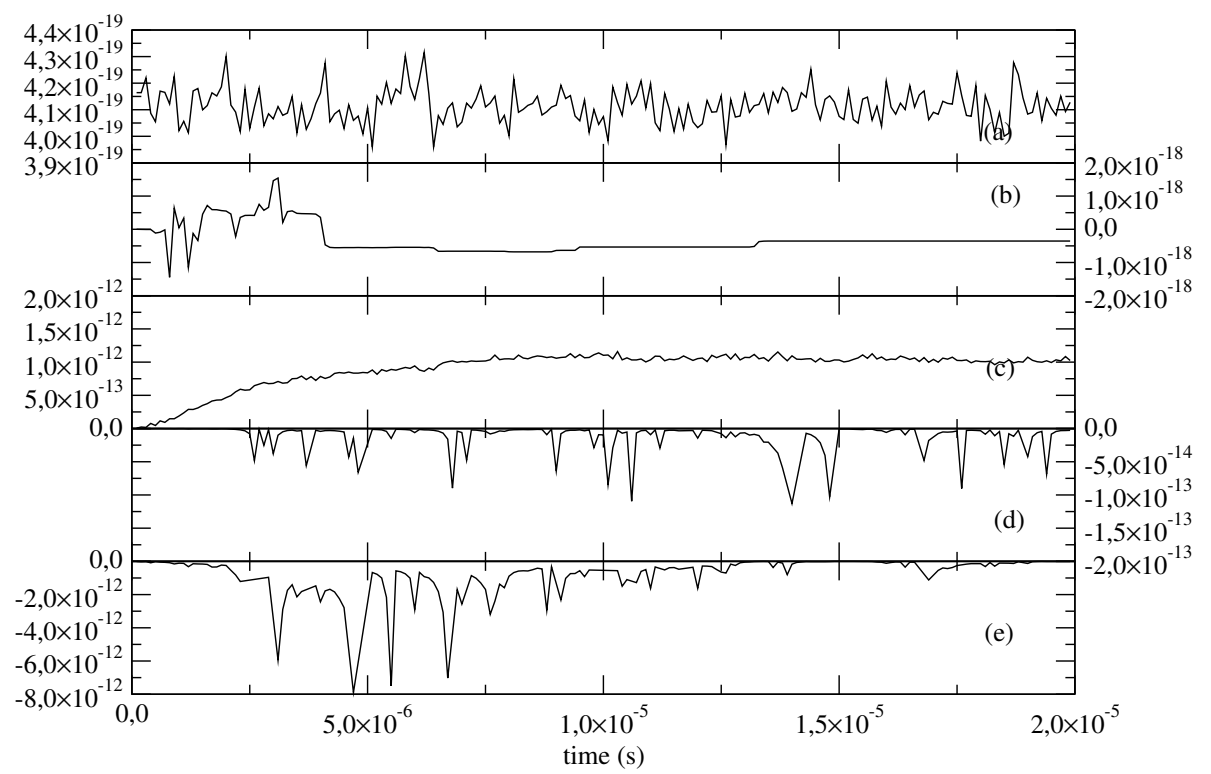

Figure 8. Plots of the mean forces per particle as a function of time when the substrate contain a deep channel bordered with rough areas (x-axis are in $s, y$-axis are in $N)$. (a) Brownian force, (b) Stokes force, (c) diffuse layer force (DLVO), (d) Van der Waals force (DLVO) and (e) capillary force. 


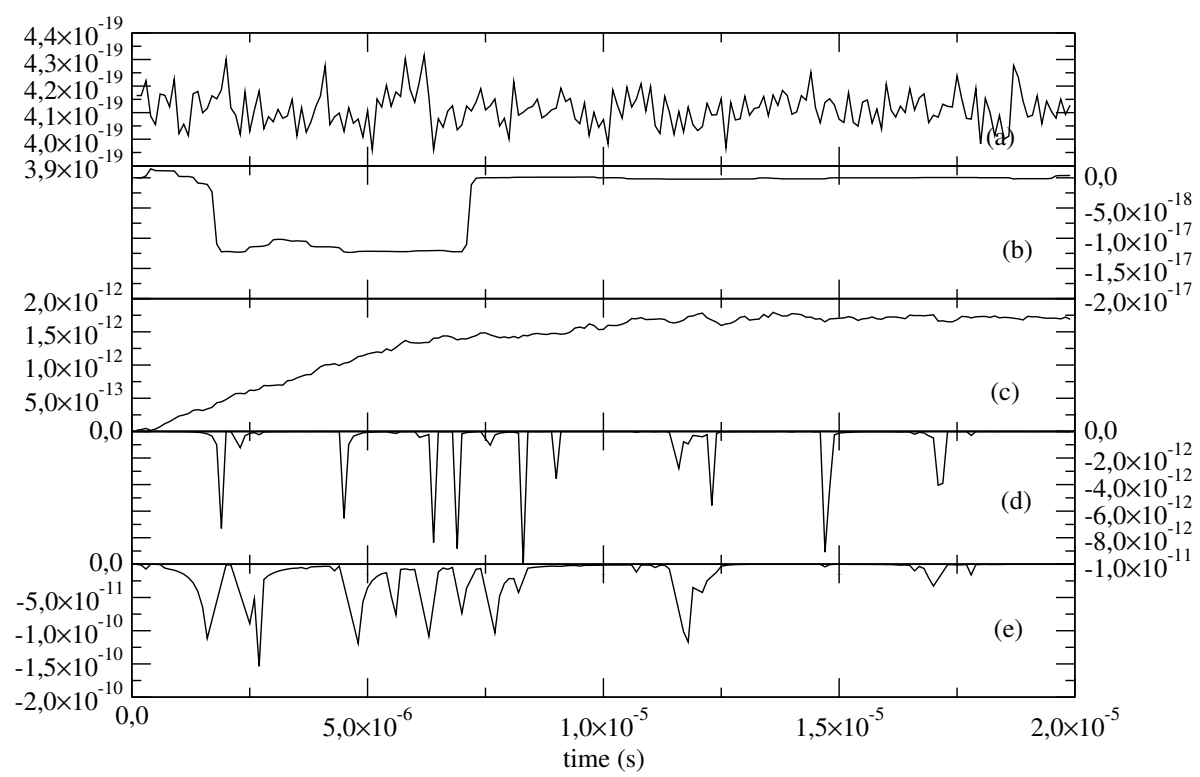

Figure 9. Plots of the mean forces for each particle as a function of time when the substrate contains two straight rough areas (x-axis are in s,y-axis are in $N$ ). (a) Brownian force, (b) Stokes force, (c) diffuse layer force (DLVO), (d) Van der Waals force (DLVO) and (e) capillary force.

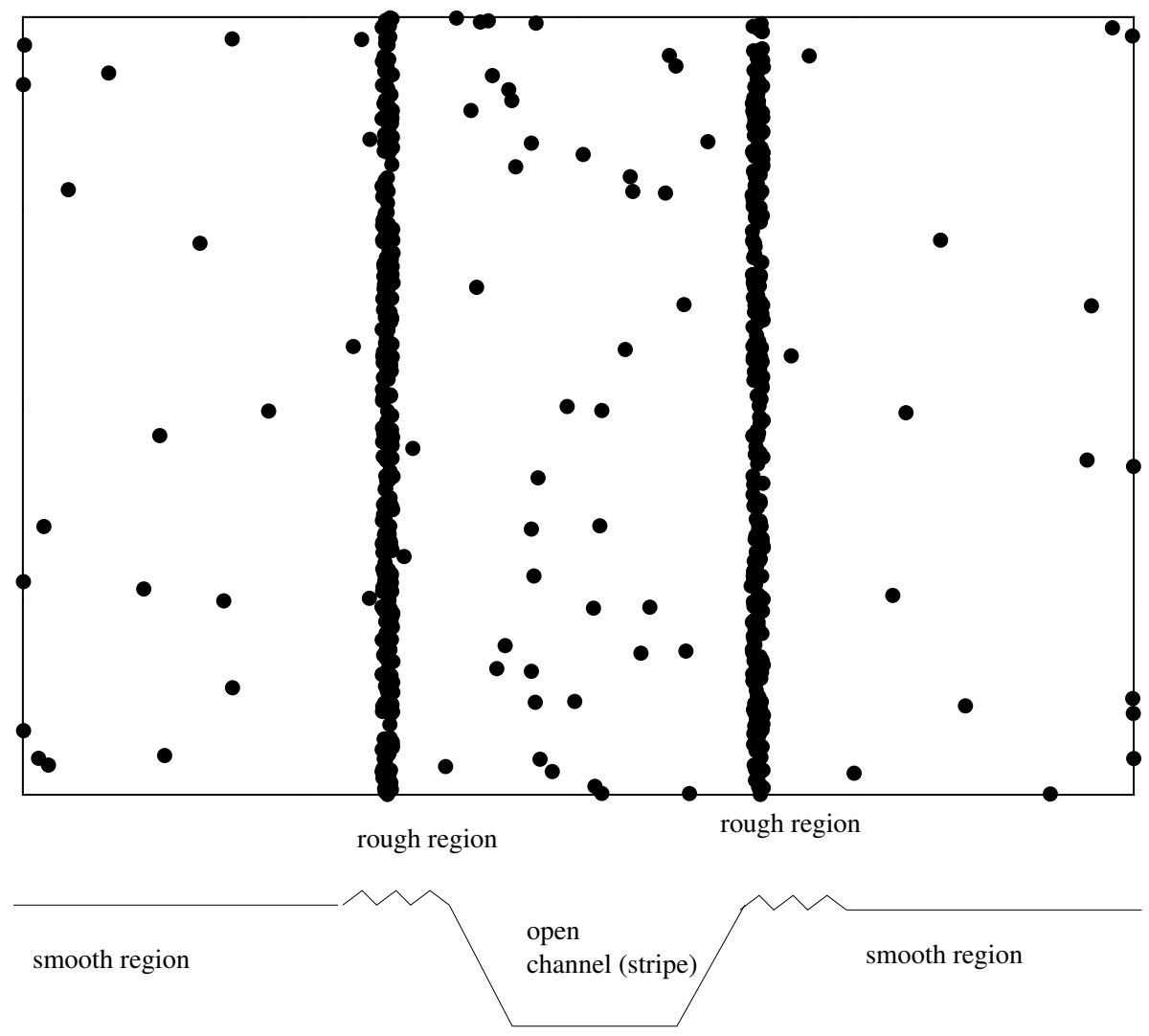

Figure 10. (top) Top view of the patterns obtained numerically on a substrate with a deep channel bordered with rough areas. (bottom) Vertical section of the substrate showing the rough areas and the open channel. 


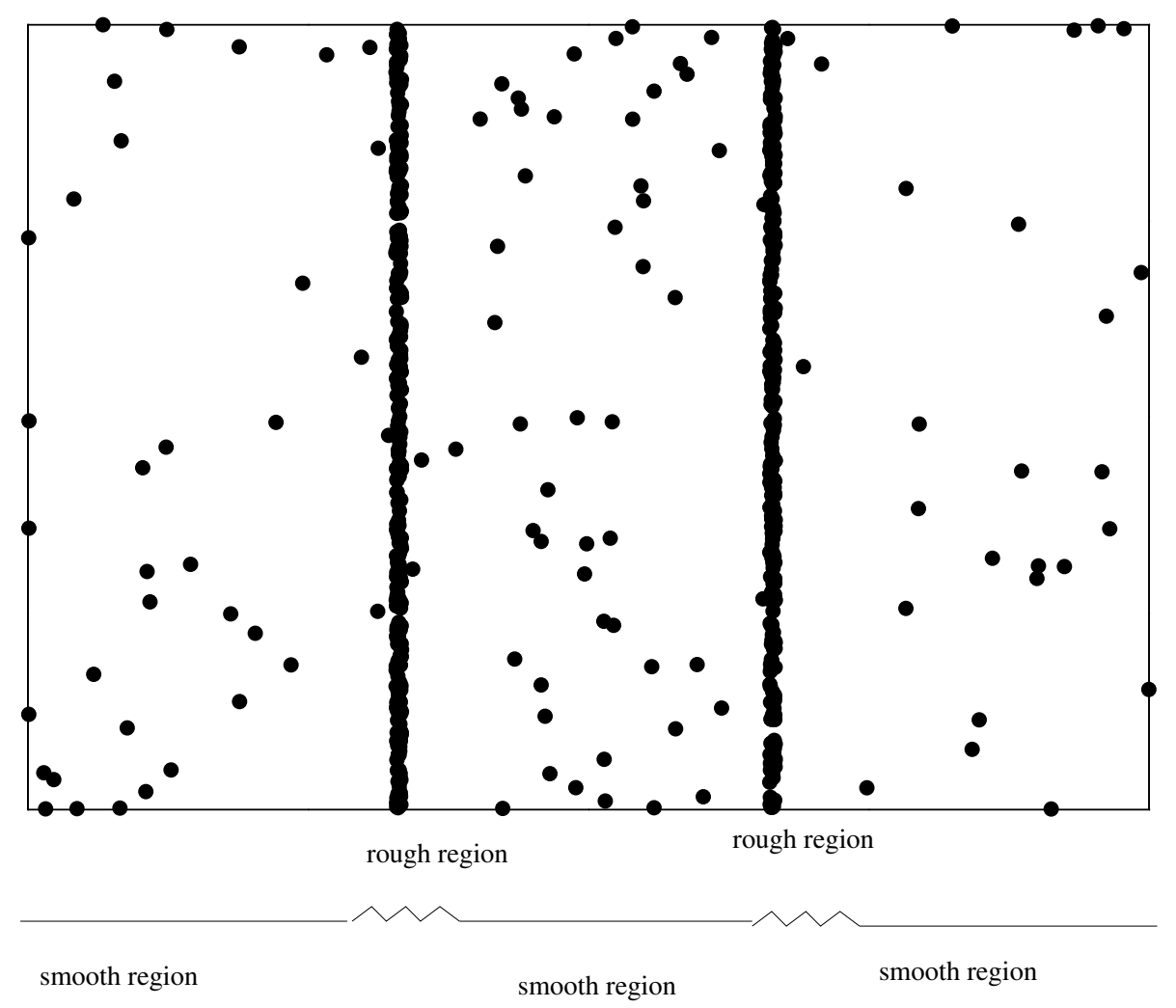

Figure 11. (top) Top view of the patterns obtained numerically on a substrate with two stripes of larger roughness. (bottom) Vertical section of the substrate showing the rough areas.

In the present study, we gave only one value to each parameter $r_{c}, \phi_{c}$ and $\alpha$ (see Figure 5 and Equations (6)-(8)). It is possible to change the values of these parameters by varying the $p H$, adding surfactants to the suspension and functionalizing the particles: the contact angle (at the air water interface) changes with the $p H$ [20], and functionalizing the PS particles or adding surfactants to the suspension also influences the capillary forces [21]. Moreover, a higher $p H$ induces increased electrostatic repulsion between particles [21] (see Equations (3) and (5) in which the electrostatic repulsion is calculated).

The sizes of the particles (micro or nanoscale) or the polydispersity of the particles influence the self-patterning [22,23]. Indeed, the particles' diameters $d$ act in Equations (1) to (8); thus, the sizes of the particles are of great importance in self-patterning processes in general, and in particular in the action of roughness (see Equation (11), which takes the sum of all the vertical forces into account, where this sum is multiplied by the kinetic friction coefficient and compared to the sum of all horizontal forces).

The patterns obtained at the end of the drying of the liquid suspension may be analyzed using various methods [24]. However, in our case, the geometrical analysis of the experimental results (see Figures 6 and 7) is difficult because of the impossibility of distinguishing between different particles.

Finally, when dealing with our modeling and in both cases (a substrate with an open channel or substrate without open channel), the vertical forces $\left(5.14 \times 10^{-11} \mathrm{~N}\right)$ are larger than the mean horizontal forces (see Figures 8 and 9). In comparison with [7], which is similar to the present study (but with faceted particles of size $10 \mu \mathrm{m}$ ), we find that particles are trapped in the rough areas, as in [7]. However, in [7], only part of the forces is computed compared with the present study. Indeed, Lallet et al. [7] calculated only the Stokes forces, the capillary forces and the Brownian forces; moreover, the friction effect was modeled by systematically multiplying the horizontal forces by 0.1 when the corresponding particle was on a rough area (without calculating the relative norms of vertical and horizontal forces). In this study, the vertical optical forces are dominating, and therefore Coulomb's law applies when computing the horizontal forces (see Equation (11)). Thus, the optical pressure has a 
non-negligible effect on the self-patterning of colloidal suspensions. Therefore, one has to take into account the spectral irradiance of the light bulb of the optical microscope when studying particles with sizes close to the light's wavelength.

\section{Conclusions}

We performed experiments regarding the drying of a horizontal suspension of micrometer-sized colloidal particles on a substrate with rough areas for the first case and with rough areas and an open channel for the second case. The horizontal width of the suspension was much larger than the width of the optical beam coming from the optical microscope; thus, we developed the hypothesis that the surface of the fluid would be horizontal and parallel to the substrate and that the contact line (between the substrate and the liquid) would be far from the optical beam.

To model these experiments, we used a Molecular Dynamics program; we took into account all the forces (capillary forces, Brownian forces, Stokes forces, DLVO forces-van der Waals forces and electrostatic double layer forces-optical forces, friction forces and weight). We did not neglect any force. The effect of evaporation has been also modeled with the decreasing depth of the liquid film: this depth acts on capillary forces, Stokes forces and Brownian forces.

We present the following conclusions:

- The patterns obtained experimentally were the same as the patterns obtained numerically;

- We computed all possible forces acting on these colloidal particles;

- We tried to model correctly the process of the drying of dispersions in the domain of particle depositions;

- We calculated the optical pressure applied on particles: this pressure cannot be neglected in our case (where the wavelength of the light coming from the light bulb of the optical microscope is close to the size of the particles);

These four facts are the novel contributions of our study.

Author Contributions: Conceptualization, L.B. and P.E.; Writing-original draft, N.O.-T. All authors have read and agreed to the published version of the manuscript.

Funding: This research and the APC were funded by 'Plateformes regionales de recherche et d'innovation 2020' of Region Occitanie (France).

Institutional Review Board Statement: Not applicable.

Informed Consent Statement: Not applicable.

Data Availability Statement: Not applicable.

Acknowledgments: All the authors thank the Opto Fluidic Platform of Montpellier (POMM) for providing the experimental results necessary to the writing of this article.

Conflicts of Interest: The authors declare no conflict of interest.

\section{References}

1. Liang, Y.; Hilal, N.; Langston, P.; Starov, V. Interaction forces between colloidal particles in liquid: Theory and experiment. Adv. Coll. Interface Sci. 2007, 134, 151-166. [CrossRef]

2. Bordin, J.R. Distinct aggregation patterns and fluid porous phase in a 2D model for colloids with competitive interactions. Physica A 2018, 495, 215-244. [CrossRef]

3. Ghosh, U.U.; Chakraborty, M.; Bhandari, A.B.; Chakraborty, S.; DasGupta, S. Effect of Surface Wettability on Crack Dynamics and Morphology of Colloidal Films. Langmuir 2015, 31, 6001-6010. [CrossRef]

4. Bevan, M.A.; Prieve, D.C. Direct Measurement of Retarded van der Waals Attraction. Langmuir 1999, 15, 7925-7936. [CrossRef]

5. Thiele, U. Patterned deposition at moving contact lines. Adv. Colloid Interface Sci. 2014, 206, 399-413. [CrossRef]

6. Giorgiutti-Dauphiné, F.; Pauchard, L. Striped patterns induced by delamination of drying colloidal films. Soft Matter 2015, 11, 1397-1402. [CrossRef] [PubMed]

7. Lallet, F.; Olivi-Tran, N. Micrometer sized particles in a two-dimensional self-assembly during drying of liquid film. Phys. Rev. E 2006, 74, 061401. [CrossRef] 
8. Aizenberg, J.; Braun, P.V.; Wiltzius, P. Patterned Colloidal Deposition Controlled by Electrostatic and Capillary Forces. Phys. Rev. Lett. 2000, 84, 2997. [CrossRef] [PubMed]

9. Pnueli, D.; Gutfinger, C. Fluid Mechanics; Cambridge University Press: Cambridge, UK, 1997.

10. McCartney, L.N.; Levine, S. An Improvement on Derjiaguin's Expression at Small Potentials for the Double Layer Interaction Energy of Two Spherical Colloidal Particles. J. Colloid Interface Sci. 1969, 30, 345-354. [CrossRef]

11. Ashkin, A. Acceleration and trapping of particles by radiation pressure. Phys. Lett. 1970, 24, 156. [CrossRef]

12. Courtesy of Keyence Research and Development.

13. Lebedew, P. Untersuchungen über die Druckkräfte des Lichtes. Ann. Der Phys. 1901, 6, 433-458. [CrossRef]

14. Nichols, E.F.; Hull, G.F. The Pressure due to Radiation. Astrophys. J. 1903, 17, 315-351. [CrossRef]

15. Alvo, S.; Lambert, P.; Gauthier, M.; Regnier, S. A van der Waals Force-Based Adhesion Model for Micromanipulation. J. Adhes. Sci. Technol. 2010, 24, 2415-2428. [CrossRef]

16. Chhasatia, V.H.; Sun, Y. Interaction of bi-dispersed particles with contact line in an evaporating colloidal drop. Soft Matter 2011, 7, 10135. [CrossRef]

17. Krachelvsky, P.A.; Nagayama, K. Capillary interactions between particles bound to interfaces, liquid films and biomembranes. Adv. Coll. Interface Sci. 2000, 85, 145-192. [CrossRef]

18. Wong, T.S.; Chen, T.H.; Shen, X.; Ho, C.M. Nanochromatography Driven by the Coffee Ring Effect. Anal. Chem. 2011, 83, 1871-1873 [CrossRef] [PubMed]

19. Visser, J. On Hamaker constants: A comparison between Hamaker constants and Lifshitz-van der Waals constants. Adv. Coll. Interface Sci. 1972, 3, 331-363. [CrossRef]

20. Vogel, N.; de Viguerie, L.; Jonas, U.; Weiss, C.K.; Landfester, K. Wafer-Scale Fabrication of Ordered Binary Colloidal Monolayers with Adjustable Stoichiometries. Adv. Funct. Mater. 2011, 21, 3064-3073. [CrossRef]

21. Vogel, N.; Goerres, S.; Landfester, K.; Weiss, C.K. A Convenient Method to Produce Close- and Non-close-Packed Monolayers using Direct Assembly at the Air-Water Interface and Subsequent Plasma-Induced Size Reduction. Macromol. Chem. Phys. 2011, 212, 1719-1734. [CrossRef]

22. Vogel, N.; Ally, J.; Bley, K.; Kappl, M.; Landfester, K.; Weiss, C.K. Direct visualization of the interfacial position of colloidal particles and their assemblies. Nanoscale 2014, 6, 6879-6885. [CrossRef]

23. Lotito, V.; Zambelli, T. Self-Assembly of Single-Sized and Binary Colloidal Particles at Air/Water Interface by Surface Confinement abnd Water Discharge. Langmuir 2016, 32, 9582-9590. [CrossRef] [PubMed]

24. Lotito, V.; Zambelli, T. Pattern detection in colloidal assembly: A mosaic of analysis techniques. Adv. Coll. Interf. Sci. 2020, 284, 102252. [CrossRef] [PubMed] 